\title{
Vers une démocratie environnementale attentive aux processus écologiques qui permettent au cycle de la vie de se perpétuer
}

\author{
Fabien Jakob*
}

Géographie, Université Laval, Centre de recherche en aménagement et développement, Québec, Canada

\begin{abstract}
Résumé - À partir d'une enquête du sens commun (Dewey, 1993), cette étude entend identifier et analyser, en prenant appui sur la sociologie de la justification (Boltanski et Thévenot, 1991), les critères de jugement et les sens de justice des acteurs engagés dans divers «forums hybrides » (Callon et al., 2001). Durant un tel processus participatif et délibératif, en l'occurrence lors de la révision du Schéma d'aménagement et de développement de l'agglomération de Québec (2015-2020), ils peuvent être amenés à repenser les systèmes de valeurs orientant les actions publiques en matière de développement territorial.
\end{abstract}

Mots-clés : aménagement du territoire / délibératif / justice / vulnérabilité / écologie

\begin{abstract}
Towards an environmental democracy attentive to the ecological processes that perpetuate the life cycle. Following a common sense based inquiry (Dewey, 1993), this study uses tools from the sociology of justification (Boltanski and Thévenot, 1991) to identify and analyse the judgement criteria and the senses of justice of actors, legitimised by their engagement in various hybrid forums (Callon et al., 2001). In the course of this participatory and deliberative process, in our case the revision of the Quebec land use planning and development scheme (2015-2020), these criteria may cause them to reconsider the value chains guiding public action in matters of territorial development.
\end{abstract}

Keywords: land use planning / deliberative / justice / vulnerability / ecology

\section{L'aménagement du territoire : le tournant participatif et délibératif}

L'aménagement du territoire entraîne des choix collectifs qui portent sur des enjeux spatiaux, sociaux, culturels, économiques et environnementaux. Il opère ainsi une mise en ordre à travers l'espace d'entités, d'équipements et d'activités en vue d'atteindre certains objectifs (exploiter, habiter, échanger, s'approprier, lutter contre la dégradation des cadres de vie, réduire les disparités, etc. ${ }^{1}$ ). Selon une approche traditionnelle ( $\ll$ top down »), il procède d'actions et de politiques publiques conduites par des élus et des experts qui interagissent dans un univers politico-administratif et technocratique, centralisé et hiérarchisé. Déterminé par des lois et règlements, des programmes, des plans, ce modèle

\footnotetext{
*Auteur correspondant : fabienjakob@bluewin.ch

${ }^{1}$ Cette recherche est conduite dans le cadre de mes activités doctorales au sein du Centre de recherche en aménagement et développement de l'Université Laval (département de géographie) au Canada.
}

traditionnel porte un regard global, rationnel, scientifique et surtout quantitatif sur la planification. À partir des années 1990, alors que les théories des mouvements sociaux mettent l'accent sur une démocratisation de la gestion publique du territoire, ce modèle, sous la triple influence d'un recadrage du débat public sur les enjeux de développement local, d'une décentralisation des actions et politiques publiques, du développement de formes renouvelées de coordination, de contractualisation et de régulation collectives à l'échelon local (déconcentration des pouvoirs), est progressivement remplacé par un processus décisionnel ascendant caractérisé par la recherche d'une vision commune du territoire. Son aménagement paraît désormais indissociable du registre de la citoyenneté de proximité, les acteurs locaux étant les mieux placés pour évaluer les besoins en infrastructures et services publics, pour aménager et pour gérer leur espace de vie. Selon des modalités plus ou moins larges et ouvertes (participation libre, tirage au sort, désignation, etc.), une part croissante des collectivités locales expérimente ainsi des dispositifs de sensibilisation et d'intéressement civique qui visent à 
corriger les imperfections du modèle représentatif par une forme de démocratie régénérée par la participation citoyenne à l'expression et à l'action politique (assemblées publiques, conseils de quartier, audiences publiques, comités consultatifs, etc.).

\section{Publicisation et problématisation de la révision du Schéma d'aménagement et de développement de l'agglomération de Québec}

Le Schéma d'aménagement et de développement de l'agglomération de Québec se présente comme un document d'orientation établissant les lignes directrices de la gestion d'un territoire dont l'objectif principal consiste en l'occurrence à soutenir le développement (industries, commerces, bureaux) de l'agglomération en ce qu'il tend à stimuler la croissance du marché immobilier en vue de favoriser l'accueil et l'installation de nouveaux acteurs aussitôt impliqués dans des interactions et coordinations constitutives de nouveaux savoirs susceptibles d'aiguiser une forme de compétitivité entre innovation, recherche scientifique et technologique participant de l'épanouissement d'un certain esprit du capitalisme (Boltanski et Chiapello, 1999).

Sa révision s'appuie sur un processus de publicisation sous la responsabilité d'une Commission consultative formée de représentants politiques des villes de Québec, de L'Ancienne-Lorette et de Saint-Augustin-de-Desmaures. Diverses campagnes de sensibilisation et de problématisation sont ainsi mises en œuvre, comme la diffusion d'articles dans la presse (notamment dans le Bulletin municipal) et l'organisation de conférences de presse (le 5 octobre 2015, le 27 avril 2016 et le 25 avril 2017). La ville de Québec considérant que les nouveaux médias participent de l'élaboration d'une opinion publique, diverses actions de communication sont également entreprises sur le Web : une courte animation est mise en ligne sur YouTube, un sondage est mis en circulation (du 8 octobre au 22 novembre 2015) auquel 203 personnes répondent ${ }^{2}$ et un webinaire est tenu le 25 mai 2016 auquel assistent 86 personnes en direct et 373 en différé (en date du 2 novembre 2017). La mise en débat se développe encore à l'occasion des séances publiques d'information ouvertes les 9 et 12 mai 2016 (webdiffusées en direct et disponibles en ligne en différé ; la séance du 12 mai est consultée 633 fois en date du 2 novembre 2017) et les 9 et 11 mai 2017 (webdiffusées en direct, visionnées en différé près de 450 fois en date du 2 novembre 2017). La Commission consultative sur le Schéma d'aménagement et de développement de l'agglomération de Québec ouvre encore des séances

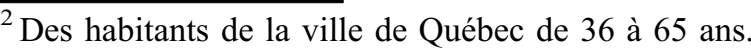

d'audition des opinions les 14,16 et 17 juin 2016 et les 29,30 et 31 août 2017 ; elles sont également diffusées sur le Web en direct et rendues disponibles en ligne en différé. L'occasion est ainsi donnée à une diversité d'acteurs de faire valoir leurs positions dans le respect de la procédure de la démarche consultative. Certains acteurs-clés (experts et techniciens, réseaux interorganisationnels, etc.) construisent leur argumentation autour d'enjeux environnementaux, comme en témoignent les revendications militantes de représentants des milieux écologiques (10 mémoires particulièrement bien articulés et argumentés sont produits dans le cadre de la consultation), auxquelles se joignent les engagements de citoyens (2 mémoires de collectifs de citoyens, 18 mémoires de citoyens intervenant à titre individuel), d'élus (2 mémoires), de représentants des milieux agricoles (2 mémoires), d'associations de quartiers ( 1 mémoire) et de lobbys immobiliers $\left(3\right.$ mémoires $\left.^{3}\right)$. Mobilisant des stratégies de dénonciation et de rupture mais également des contre-propositions très informées, plusieurs de ces mémoires s'opposent en particulier au dézonage des terres des Sœurs de la Charité vendues au fonds de placement immobilier Cominar, créé en 1998 par Jules Dallaire qui devient ainsi le plus grand acquéreur de terres foncières agricoles ${ }^{4}$, leur valorisation étant confiée à la société en commandite Terres d'espérance, qui espère générer un profit de 150 millions de dollars canadiens. La critique dénonce la nécessité de construire de nouveaux logements sur ces espaces verts périurbains indispensables à la qualité de vie citadine en contestant la précision des perspectives de croissance démographique et la pertinence de certains indicateurs (critique du concept de ménage en faveur d'une planification basée sur les groupes d'âges, etc.). Plusieurs argumentaires soulignent également une incompatibilité entre la réaffectation de certaines parcelles à vocation agricole et la volonté politique affirmée d'assurer la pérennité d'activités agricoles prospères. Ces différents arguments sont résumés dans des rapports de consultation à partir desquels sont élaborés le premier et le second projet de Schéma d'aménagement et de développement révisé de l'agglomération de Québec.

\footnotetext{
${ }^{3}$ Ces mémoires sont disponibles à l'adresse https://www.ville. quebec.qc.ca/apropos/planification-orientations/amenagemen t urbain/sad/memoires.aspx.

${ }^{4}$ L'acquisition par un fonds de placement coté en Bourse permet de regrouper au sein d'une structure d'investissement un certain nombre d'actionnaires plus ou moins anonymes. Cela permet également de contourner l'application de la loi sur l'acquisition des terres agricoles par des non-résidents qui interdit l'acquisition de terres agricoles par des personnes physiques ne résidant pas au Québec (les fonds d'investissement ne sont pas visés par ces dispositions).
} 


\section{Engagements civiques et contre-mobilisations citoyennes}

Le projet de dézoner les Terres d'espérance, soit la perte irréversible d'une terre agricole patrimoniale (il faut près de 5000 à 7000 ans pour constituer $40 \mathrm{~cm}$ de sol arable), provoque également la constitution de micropublics concurrents et de publics de plus grande envergure (Dewey, 2010), dans des espaces publics d'expression et de revendication relativement indépendants des agents de programmation et de régulation de l'ordre politique, soit la mobilisation d'un ensemble d'acteurs, d'organisations et d'institutions, indirectement concerné par la perception partagée des conséquences indésirables d'une situation problématique (Cefaï et al., 2012) et qui s'implique pour tenter d'y faire face.

Les engagements délibérés, les initiatives ascendantes, les rassemblements plus circonstanciels, réunissant des lanceurs d'alerte empruntant des modalités réactualisées d'action politique, quelques élus ${ }^{5}$, des représentants d'associations patrimoniales, de milieux écologiques et agricoles, rompant (pour certains) avec le militantisme dans sa forme traditionnelle (parti), ainsi que des citoyens plus ou moins ordinaires explorant d'autres façons de résister (Rosanvallon, 2006), empruntent différentes modalités: l'appel aux citoyens de Québec (décembre 2014) de l'Union des producteurs agricoles de la Capitale-Nationale-Côte-Nord pour demander le maintien en zone agricole de ces terres patrimoniales ; le sondage réalisé en juin 2016 relatif à la volonté citoyenne de protéger les terres agricoles; plusieurs interventions médiatiques; le forum «Une journée pour la terre» du 9 novembre 2015; la mobilisation le 10 juin 2017 de près de 200 citoyens et agriculteurs le long des rues du secteur agricole de l'arrondissement Beauport en signe d'opposition au dézonage de ces terres; le rassemblement «Rêvons les terres des Sœurs de la Charité » organisé le 3 novembre 2019 par Voix Citoyenne, etc. Aux pratiques de «militantisme post-it», caractérisées par un certain individualisme et l'usage des réseaux sociaux, s'ajoutent des scènes alternatives de la parole publique souvent en lien avec des «médiations (ce par quoi il faut passer) médiatiques et technologiques » (Granjon, 2014, p. 98), des espaces de production, de collecte, de diffusion et de circulation d'informations. Les acteurs identifient, confrontent, codéfinissent des problématiques dans l'interactivité d'un flux communicationnel qui façonne, transforme et traduit une opinion publique projetée de microréseaux personnels dans des macroréseaux et dont l'écho est susceptible de court-circuiter, contrer, voire discréditer, certains discours politiques. Internet et la dimension participative du Web 2.0 permettent à cet

\footnotetext{
${ }_{5}^{5}$ Démocratie Québec, Parti québécois.
}

égard non seulement de transformer la dyade public/ privé, d'élargir l'espace public mais encore de faciliter une forme de réappropriation du débat public.

En s'opposant à la périurbanisation, en voulant freiner le développement territorial et préserver une certaine qualité du cadre de vie, les critiques du projet d'aménagement visent la logique industrielle et technocratique qui le sous-tend, proches en cela de la cause écologique des années 1970. Mais d'autres contestations dénoncent les violations de lois et règlements (notamment la loi sur le patrimoine culturel, la loi sur la protection du patrimoine naturel et la loi sur la protection du territoire et des activités agricoles), tout en avançant des témoignages fondés sur des expériences de terrain ou en produisant des informations proprement scientifiques. La critique mobilise également un mode d'évaluation d'ordre esthétique, en particulier en référence au paysage, mais également le respect des lieux, l'attachement au territoire, l'attention portée au passé qui attestent de «l'actualité d'un principe de justification fondé sur le respect de la tradition et la grandeur d'un lien de confiance de nature domestique » (Lafaye et Thévenot, 1993, p. 505).

\section{Une prise de conscience (plus ou moins) collective de l'importance des Terres d'espérance}

Les Terres d'espérance offrent une vue panoramique exceptionnelle sur le fleuve Saint-Laurent, la rive sud et l'île d'Orléans; à ce titre, elles représentent un bien commun qui ne peut être confisqué par quelques particuliers. Objet de nature certes, mais aussi lieu d'une intervention humaine qui concerne l'architecture végétale mais aussi d'autres constructions plus artificielles (délimitations du terrain, implantation et orientation des édifices, aménagement des espaces extérieurs en fonction d'éléments naturels, etc.). Non seulement ce territoire représente la plus ancienne trame agricole du pays $^{6}$, mais il héberge un ensemble bâti religieux - lieux de culte, bâtiments conventuels -, ainsi que tous les biens matériels, les objets de culte, les tableaux et sculptures aux sujets religieux et les milliers d'archives que ces bâtiments contiennent ${ }^{7}$. Est mis en question le devenir du patrimoine que les Sœurs de la Charité vieillissantes et numériquement en déclin se résolvent à vendre, à l'instar

\footnotetext{
${ }^{6}$ Faisant partie de la seigneurie de Beauport concédée en 1634 à Robert Giffard par la Compagnie des Cent-Associés, les Terres d'espérance sont rachetées par lots dès la fin du $\mathrm{XIX}^{\mathrm{e}}$ siècle par les Sœurs de la Charité.

${ }^{7}$ Les Sœurs de la Charité de Québec ont toutefois déjà fait don au Musée de la civilisation et à d'autres musées de la plus grande partie de leurs collections (3000 entrées); seules 600 entrées sont retenues, laissant ouverte la question de la sauvegarde du patrimoine religieux.
} 
de toutes les communautés religieuses du Québec qui se départissent de la lourde charge qu'imposent leurs biens immobiliers afin de subvenir à leurs besoins et assurer la poursuite de leurs œuvres.

C'est bien ce paysage culturel, c'est-à-dire ce paysage qui n'aurait pas le même aspect ni le même sens sans l'empreinte sur la terre et l'environnement laissée par les hommes, leurs activités et modes de vie, qu'entendent sauvegarder plusieurs acteurs. Leurs mobilisations participent en effet, sous l'angle d'une expérience sociale, d'un processus par lequel des acteurs pluriels entendent par leurs actions et leurs engagements conférer à certains objets (espaces architecturaux, urbanistiques ou paysagers, pratiques sociales, etc.) un ensemble de propriétés ou de «valeurs » reconnues et partagées, parce qu'ils se les sont appropriées, s'y reconnaissent, s'en soucient et en proclament l'existence. Visant de la sorte à faire reconnaître collectivement "l'importance intrinsèque (ce en quoi cet objet est représentatif d'une histoire légitime des objets de société) et extrinsèque (ce en quoi cet objet recèle des valeurs supports d'une mémoire collective)» (Lazzarotti, 2003, p. 692) de ces terres de nature et de culture, ils rendent ainsi compte «d'une relation vivante de ceux qui habitent avec leur passé» (Choay, 2006, p.322), d'une géographicité (Dardel, 1952) qui pourrait sous-tendre une forme de patrimonialisation (Berthold, 2018).

\section{Les Terres d'espérance comme support de processus écologiques permettant au cycle de la vie de se perpétuer}

$\mathrm{Si}$ cette construction paysagère procède d'une superposition mosaïque d'empreintes plus ou moins bien conservées d'une succession de phénomènes matériels et immatériels, d'une mémoire du passé humain et écologique, elle sous-tend également un processus de transformation sous l'influence successive de contraintes et/ou d'atouts naturels, sociaux et culturels faisant écho à des préoccupations non seulement passées, présentes mais encore futures; cette dimension prospective participe d'une réflexion sur les ressources (naturelles ou non) plus ou moins renouvelables ou coûteuses qu'engagent par leurs actions les acteurs à l'avenir.

En interrogeant la satisfaction des besoins alimentaires des citoyens de l'agglomération, plusieurs acteurs défendent la vocation nourricière des Terres d'espérance qui comptent parmi les plus productives du pays, d'autant plus que le secteur de l'agriculture est une pierre angulaire de l'économie et de la sécurité alimentaire du Canada, les terres propices à l'agriculture ne représentant que $5 \%$ de la superficie totale du territoire canadien ( $2 \%$ à Québec) et ayant été mises à mal au cours des dernières décennies par l'étalement urbain : de 2001 à 2010, la superficie agricole a diminué de 969802 hectares tandis que, durant la même période, la superficie des zones habitées a augmenté de 19\%. Désireux de sauvegarder ces ressources, ces acteurs envisagent non seulement des formes d'agriculture durable favorisant notamment les circuits courts d'approvisionnement moins dommageables pour l'environnement, mais avancent encore de possibles développements novateurs sur les Terres d'espérance, comme la mise en place de lieux d'expérimentation de cultures maraîchères alternatives (tables urbano-champêtres gastronomiques, autocueillette, forêts nourricières diversifiées, conception de jardins communautaires), la délimitation d'une ceinture verte incluant les 200 hectares des Sœurs de la Charité, les 120 hectares de la ferme Bédard et Blouin ainsi que les terrains forestiers acquis en 2014 par la ville de Québec (Bourg Royal). Ils proposent également l'ouverture d'un centre d'expertise en agriculture urbaine ainsi que d'autres projets d'intérêt public (notamment la constitution d'une fiducie financière agricole offrant diverses opportunités d'intégration sociale, économique et culturelle).

D'autres acteurs, écologiquement plus engagés, considèrent quant à eux non seulement les Terres d'espérance comme un cadre de vie mais surtout comme le support de processus écologiques qui permettent au cycle de la vie de se perpétuer. À ce titre, ils plaident pour un mode de faire ou de partager vertueux sous-tendu par un sens accru des responsabilités; ils en appellent à des dynamiques collectives d'association et de solidarité fondées sur la coopération, le mutualisme qui favorisent, suivant un processus de sollicitude, l'organisation et la distribution des soins dévolus à des situations caractérisées par une forme de vulnérabilité.

\section{Remarques conclusives}

À l'occasion de la révision du Schéma d'aménagement et de développement de l'agglomération de Québec, un travail d'argumentation (de scientifiques, de représentants d'associations écologiques ou agricoles, d'élus des partis de gauche et d'extrême gauche, de régionalistes, d'amis de la nature, de défenseurs de sites menacés, de pratiquants de techniques alternatives comme l'agriculture biologique, d'adeptes de la vie communautaire, etc.) a donc eu lieu au sein des arènes institutionnelles traditionnelles comme dans des espaces d'expression et d'action plus périphériques. Il fait appel, pour reprendre les catégories de la théorie des grandeurs, à la justification civique (le respect des lois), à la critique $\mathrm{du}$ modèle industriel et marchand (facteur de crise environnementale) ainsi qu'à un mode d'évaluation d'ordre inspiré (l'esthétique et le sensible). Il permet de 
faire reconnaître collectivement l'importance qu'accordent les acteurs mobilisés à ces terres de nature et de culture.

La radicalité de la critique portée dans l'arène des débats fait échouer l'adoption du premier (octobre 2018) et du second projet (novembre 2019) de Schéma d'aménagement et développement révisé de l'agglomération de Québec, celui-ci étant sanctionné par un avis de non-conformité aux orientations gouvernementales en matière d'aménagement du territoire. À partir de l'amendement de plusieurs dispositions, est entérinée le 7 février 2020 une version favorable au maintien en zone agricole des Terres d'espérance et, plus généralement, respectueuse des espaces verts et des dimensions sociales et culturelles du site. Il s'agit là d'une victoire pour les militants écologiques et représentants des milieux agricoles et patrimoniaux. Couronnés d'un double succès, leurs engagements pourraient par ailleurs déterminer un changement de pratique en matière de gestion territoriale: le gouvernement semble en effet désormais contraint d'obtenir un avis favorable de la Commission de la protection du territoire agricole (CPTA) avant d'introduire tout projet de déclassement de terres agricoles dans ses schémas et plans d'aménagement du territoire (précédemment, la validation de la CPTA était sollicitée après l'entrée en vigueur des plans et schémas). La virulence de la critique accule par ailleurs le groupe Dallaire à revendre, non sans une perte financière sérieuse, les terres aux Sœurs de la Charité, qui $s$ 'engagent à respecter la vocation nourricière de cet écrin de verdure.

\section{Références}

Berthold E., 2018. Le patrimoine des communautés religieuses. Empreintes et approches, Laval, Presses de l'Université Laval.

Boltanski L., Chiapello E., 1999. Le nouvel esprit du capitalisme, Paris, Gallimard.

Boltanski L., Thévenot L., 1991. De la justification. Les économies de la grandeur, Paris, Gallimard.

Callon M., Lascoumes P., Barthe Y., 2001. Agir dans un monde incertain. Essai sur la démocratie technique, Paris, Le Seuil.

Cefaï D., Carrel M., Talpin J., Eliasoph N., Lichterman P., 2012. Ethnographies de la participation, Participations, 4, 3, 7-48, https://doi.org/10.3917/parti.004.0005.

Choay F., 2006. Pour une anthropologie de l'espace, Paris, Le Seuil.

Dardel E., 1952. L'homme et la terre, Paris, PUF.

Dewey J., 1993. Logique. La théorie de l'enquête, Paris, PUF.

Dewey J., 2010. Le public et ses problèmes, Paris, Gallimard.

Granjon F., 2014. Citoyenneté, médias et TIC, Réseaux, 184-185, 2-3, 95-124, https://doi.org/10.3917/ res. 184.0095 .

Lafaye C., Thévenot L., 1993. Une justification écologique? Conflits dans l'aménagement de la nature, Revue française de sociologie, 34, 4, 495-524. https://doi.org/10.2307/ 3321928.

Lazzarotti O., 2003. Patrimoine, in Lévy J., Lussault M. (Eds), Dictionnaire de la géographie et de l'espace des sociétés, Paris, Belin, 692-693.

Rosanvallon P., 2006. La contre-démocratie. La politique à l'âge de la défiance, Paris, Le Seuil.

Citation de l'article : Jakob F. Vers une démocratie environnementale attentive aux processus écologiques qui permettent au cycle de la vie de se perpétuer. Nat. Sci. Soc. 29, 2, 233-237. 